\title{
Treatment of Anderson Type II Odontoid Fracture in Elderly Patients by Posterior Pedicle Screw Fixation Combined with Iliac Bone Grafting
}

\author{
Dun Liu1, Yong Wang1, Bing Hu${ }^{1 *}$, Jinjun $\mathrm{Li}^{2 *}$ \\ ${ }^{1}$ Department of Orthopedics, Tianyou Hospital Affiliated to Wuhan University of Science \& Technology, Wuhan, China \\ ${ }^{2}$ Department of Surgery, College of Medicine, Wuhan University of Science and Technology, Wuhan, China \\ Email: ^hubinghuda@163.com, *675812416@qq.com
}

How to cite this paper: Liu, D., Wang, Y., $\mathrm{Hu}$, B. and Li, J.J. (2017) Treatment of Anderson Type II Odontoid Fracture in Elderly Patients by Posterior Pedicle Screw Fixation Combined with Iliac Bone Grafting. International Journal of Clinical Medicine, 8, 572-582.

https://doi.org/10.4236/ijcm.2017.811054

Received: October 13, 2017

Accepted: November 12, 2017

Published: November 15, 2017

Copyright ( 92017 by authors and Scientific Research Publishing Inc. This work is licensed under the Creative Commons Attribution International License (CC BY 4.0).

http://creativecommons.org/licenses/by/4.0/

\section{(c) (i) Open Access}

\begin{abstract}
Objectives: To explore the treatment and related prognosis of elderly patients with Anderson II odontoid fracture with posterior pedicle screw fixation combined with iliac bone grafting. Methods: Retrospective analysis of 17 cases of elderly patients with Anderson II odontoid fracture who underwent posterior pedicle screw fixation combined with iliac bone grafting from January 2013 to December 2016. 17 patients had a history of trauma before surgery, and they all have varying degrees of atlantoaxial instability or subluxation and varying degrees of neck occipital pain and limited mobility. Result: No spinal cord or vertebral artery injury occurred during surgery. Follow-up information is complete. The follow-up period was 6 to 48 months (mean $27.4 \pm 12.4$ months). Postoperative imaging review prompted a good reduction of cervical spine, stable sequence; no pedicle screw loosening, fracture, iliac bone graft at the location of the situation, odontoid fracture and bone healing at the good, the patient after cervical rotation are limited to varying degrees. Conclusion: Posterior pedicle screw fixation combined with iliac bone grafting in elderly patients with Anderson II odontoid fracture can achieve good stability, and the prognosis is good, but long-term cervical rotation function may be affected to varying degrees.
\end{abstract}

\section{Keywords}

Anderson Type II Odontoid Fracture, Cervical Posterior Approach, Pedicle Screw Fixation 


\section{Introduction}

Cervical odontoid fracture is a more common type in the elderly patients with spine-related fractures, car accident and other violent trauma caused by elder Anderson II odontoid fracture is relatively rare. Most of the elderly Anderson II odontoid fracture is due to fall. The minor injuries caused by the injury, and the main injury mechanism are due to the patient when the injury caused by excessive neck extension. The most commonly used classification method in the world is Anderson's previous experience. The Anderson classification is proposed in the 1970s, and the improved Anderson-D'Alonzo classification is based on Grauer, which is the most common type II odontoid fracture [1]. Elderly odontoid fractures in patients with no surgical treatment, only give conservative treatment (such as strict relax on bed, line Halo-vest external fixation, etc.). The fracture healing rate of odontoid fracture can up to $50 \%-80 \%$ [2], and may be caused by the relevant serious complications due to atlantoaxial instability, so most scholars consider that the vast majority of elderly odontoid fractures need to be given surgical diagnosis and treatment [3]; but for the elderly odontoid fracture, there is still more controversy of the choice of surgical methods [4]. There are many kinds of internal fixation methods, such as posterior lateral mass screw fixation (Magerl operation), posterior lateral mass screw fixation (Apofix operation), Brooks posterior wire fixation, Halifax posterior laminectomy and so on. This article is a retrospective analysis which used posterior pedicle screw fixation combined with iliac bone grafting for elderly Anderson II odontoid fractures from January 2013-December 2016 December to explore the efficacy of fractures and related prognosis.

\section{Materials and Methods}

\subsection{General Information}

Retrospective analysis of non-obsolete (injury time $<24 \mathrm{~h}$ ) of odontoid fractures was conducted between January 2013 and December 2016.According to Anderson's classification and Grauer's modified Anderson-D'Alonzo fraction Type [5], clear diagnosis for Anderson II odontoid fracture, and posterior pedicle screw fixation combined with iliac bone graft were given in 17 patients. All patients received complete follow-up data, including 10 males and 7 females, aged $60-78$ years, mean age 67.3 years, injuries: 13 cases of injuries, 3 cases of car accident injuries, 1 case of falling injuries; Elderly patients with postoperative injury were prompted for Anderson II type odontoid fracture. All these patients have neck pain and limited cervical activity, 2 cases (11.8\%) associated with double upper limb numbness, weakness, 1 case (5.9\%) accompanied by lower limb weakness, according to ASIA spinal cord injury classification [6]: D grade 3 cases, E grade 14 cases. This study was approved by the ethics committees of our hospital, and each patient provided informed consent.

\subsection{Preoperative Assessment}

Strict relax on bed before operation, supine position pillow jaw with traction, trac- 
tion weight specific are according to the patient's weight, generally the weight were no more than $5 \mathrm{~kg}$, traction time has been maintained until the surgery. If the patient with atlantoaxial dislocation, we usually don't forced a complete reset, just pillow and jaw traction can be stable. Atlantoaxial MRI and atlantoaxial CT scan + three-dimensional reconstruction to understand the mandibular odontoid fracture, displacement and the location of the cervical spondylolisthesis, such as: cervical spine, Whether the spinal cord compression or not; vertebral artery CTA examination to confirm the feasibility of posterior pedicle screw development and to see whether the patient with vertebral artery congenital anomalies or deformities (Figure 1 and Figure 2). The cervical spine JOA score, VAS pain score, cervical spondylosis score (NDI) score were used to compare the patient's preoperative neck pain and neck rotation dysfunction.
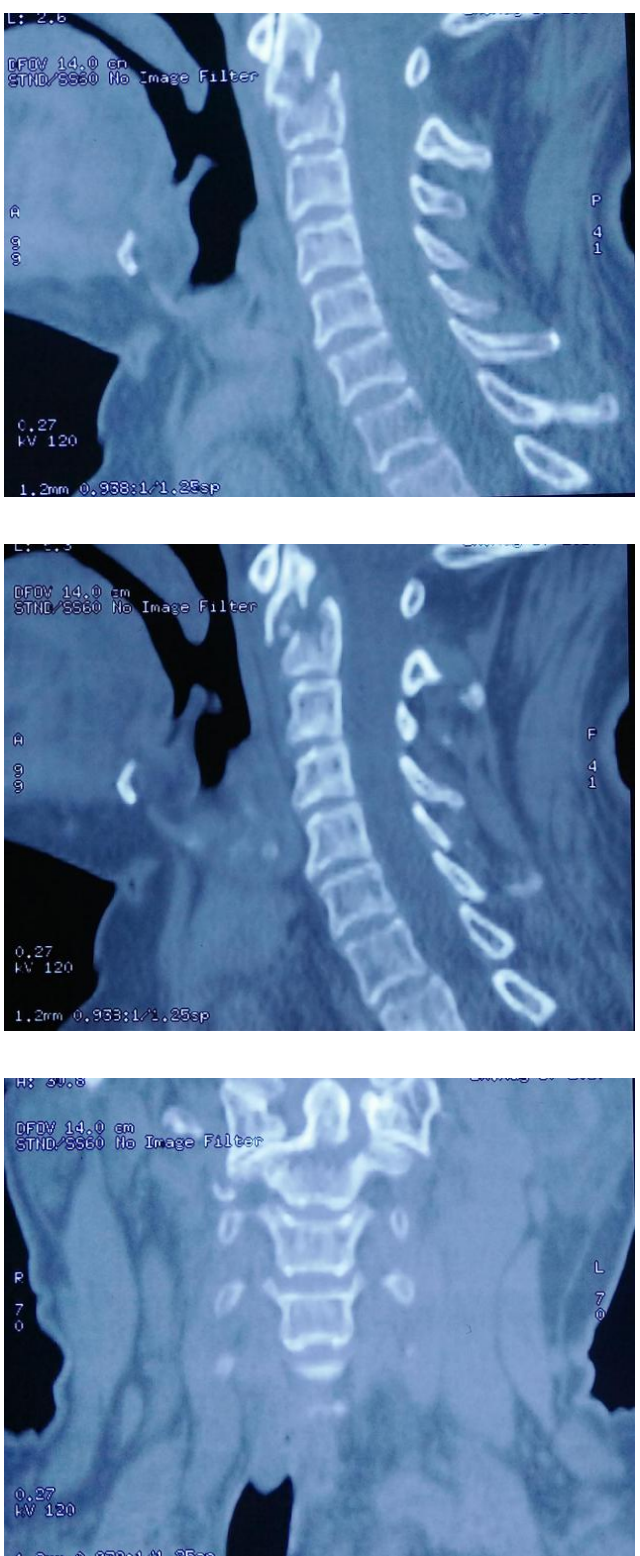

Figure 1. Preoperative CT. 

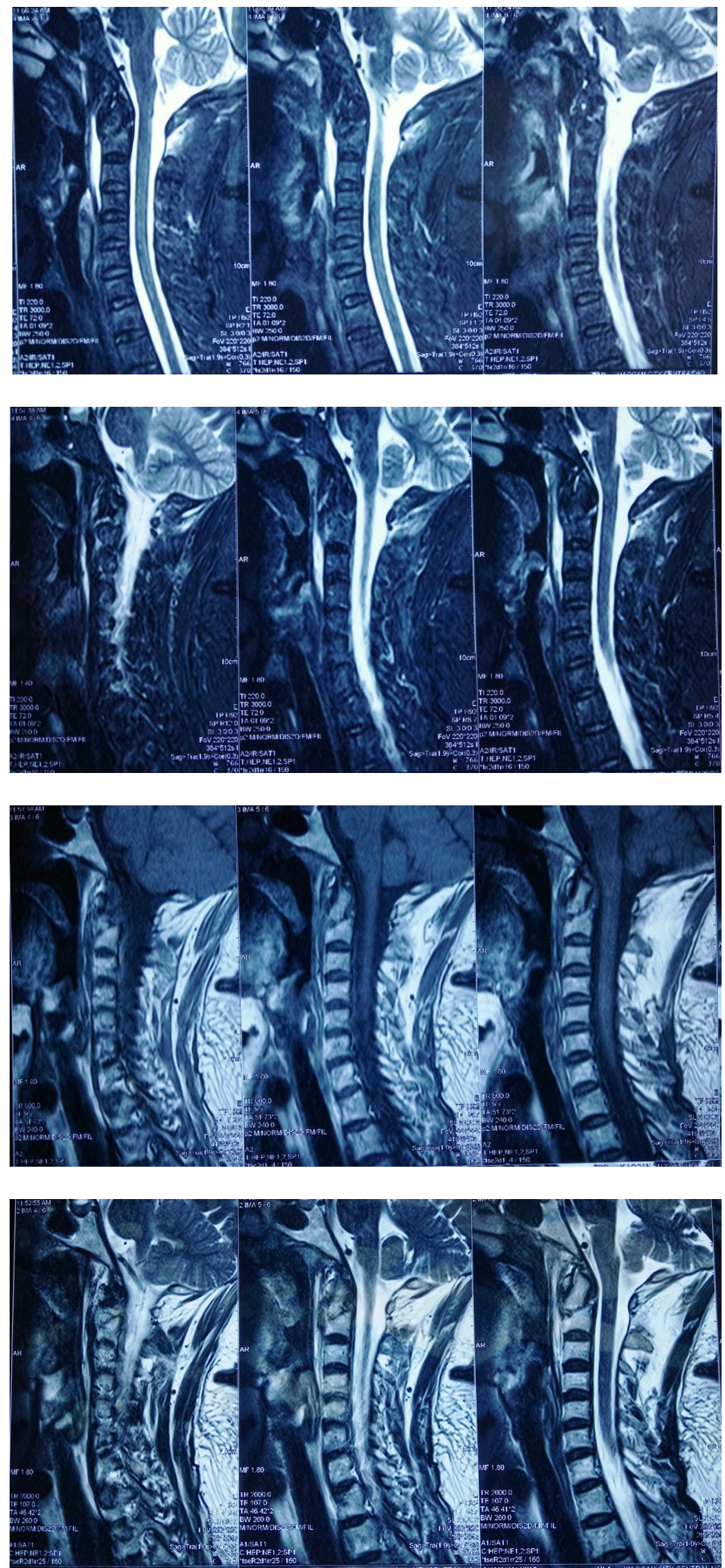


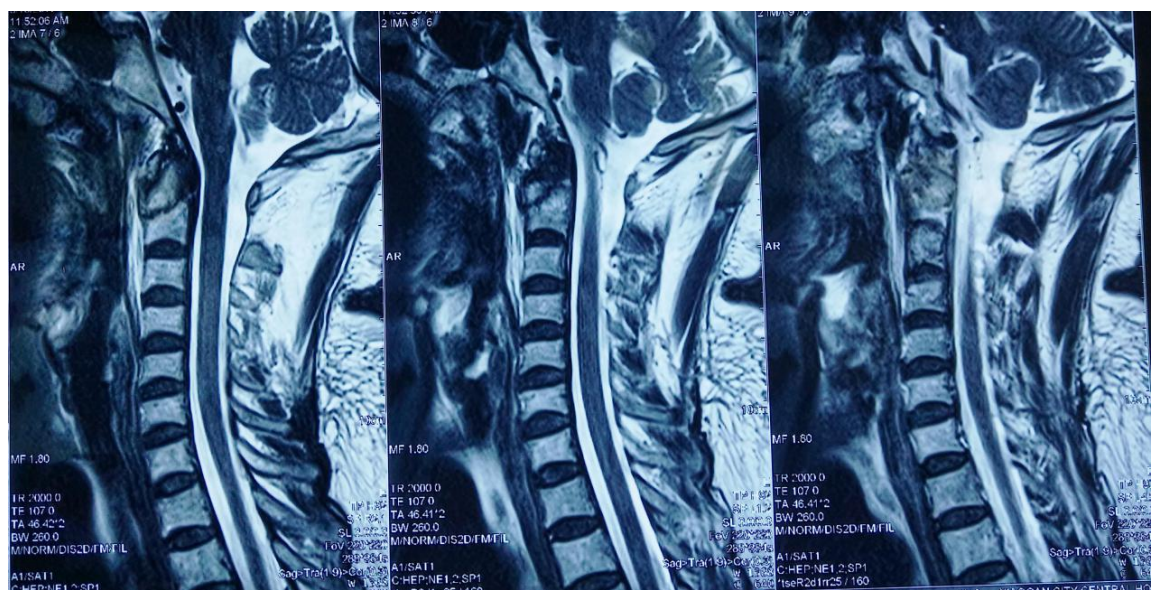

Figure 2. Preoperative MRI showed delayed odontoid fractures, according to AndersonD'Alonzo classification is divided into IIc type.

\subsection{Surgical Methods}

17 elderly patients were taken posterior bilateral pedicle screw fixation technology, both sides of the upper and lower installation of fixed rods. Among them, 14 cases were treated with C1-2 pedicle screw fixation and 3 cases were treated with $\mathrm{C} 1$ and $\mathrm{C} 3$ posterior pedicle screw fixation.

All surgeries were performed by the physician at the chief physician level. After the success of general anesthesia, the patient first take the supine position, routine disinfection shop towel at the left iliac spine, to do about $5 \mathrm{~cm}$ long incision in the left iliac spine, followed by stripping left side of the iliac bone, take about $3 \times 2 \mathrm{~cm}$ size bone Block spare. Under the wound place the appropriate drainage tube. Then take the prone position, neck department to take moderate extension, traction, the neck to maintain the neutral position. For about $8-10$ $\mathrm{cm}$ incision from the occipital neck after the middle of the occipital protrusion down, Gradually cutting the skin, fascia and ligaments, the fascia incision to the distal expansion to the third cervical spinous process, deep to occipital protuberance, carefully separated the paraspinal muscles, showing atlas and the axis, along the upper edge of the vertebral arch, isolated atlantoaxial joint capsule, pay attention to protect the pillow nerve. To prevent atlantoaxial lateral venous sinus hemorrhage, If accidentally cause bleeding, then use gelatin sponge or gauze packing to stop bleeding, in addition, it should be noted that in the exposed atlas after the arch, cannot go beyond the midline $1.5 \mathrm{~cm}$, this position through the vertebral artery ,to prevent not to hurt the vertebral artery, thus causing bleeding. Neuronal stripping probe to carefully identify the $\mathrm{C} 2$ pedicle position of the pedicle screw, $\mathrm{C} 2$ pedicle screw on the needle point in its outer quadrant, from the medial margin and C2 under the articular process of the lower edge of about $3-5 \mathrm{~mm}$. Grinding drill or sharp cone after passing through the cortical bone, $2 \mathrm{~mm}$ elastic pedicle probe path, the direction of the inside, to the head of $20^{\circ}-30^{\circ}$, just over the cortical bone. With the probe to explore the well after the wall tapping, implantation diameter of $3.5 \mathrm{~mm}$, the length of moderate (the length of the probe ac- 
cording to the depth of the probe) of the pedicle screw; and then use the nerve stripping off the atlas and interphalangeal joint Capsule, ensure the separation, in the atlas after the bow and its side of the continuation of the site of the middle point slightly lower, that is, atlas pedicle screw into the needle point, use the tools to break the bone cortex, with pedicle probe Direction, direction for the front and rear bit down or slightly inward, sagittal parallel to the rear arch and aiming at the front of the front arch, toward the inside into the angle of about $10^{\circ}$, should be just over the anterior cortex. Use the probe to detect, confirm the integrity, tapping $3.5 \mathrm{~mm}$ screws after implantation. Install the diameter of 3.5 $\mathrm{mm}$ pre-bent peptide fixation rods in the screw, atlas, after the reduction of the screw locking screw.C3 posterior pedicle screw implantation method: use the Magerl method of implantation of screws, the needle point in the side of the central point of the center of $2 \mathrm{~mm}$, drilling direction for the extrapolated $20^{\circ}-25^{\circ}$, head tilt $30^{\circ}-40^{\circ}$. With a diameter of $2.5 \mathrm{~mm}$ drill punch hole, the depth of 12 $18 \mathrm{~mm}$. Probe direction, tapping after pedicle screw implantation. Take the autologous iliac bone, $\mathrm{C} 1, \mathrm{C} 2$ or $\mathrm{C} 1-\mathrm{C} 3$ bone graft bone graft after implantation, laminectomy and atlantoaxial interbody fusion fusion (Figure 3). Strict bed rest after surgery, the third day after the surgery, remove the neck wound drainage tube, wearing neck care in the case of getting out of bed activities, neck wear strict wear a month. Postoperative X-ray film review every week one month after surgery, and then review the X-ray film every month, according to the results of the review to determine the specific removal of the nest time and the next specific functional exercise program.
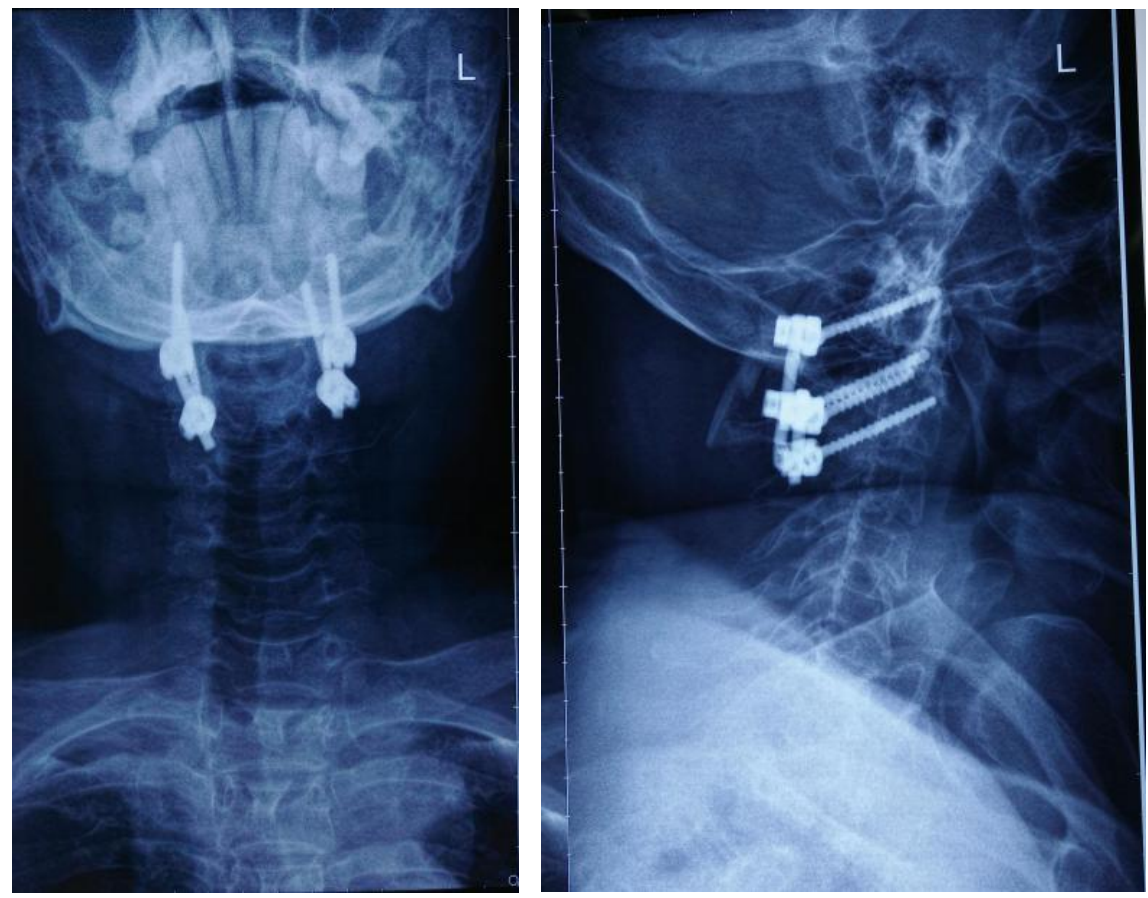

Figure 3. Posterior pedicle screw fixation combined with iliac bone graft after X-ray showed pedicle screw fixation, iliac bone graft at a good location. 


\subsection{Statistical Methods}

Using SPSS20.0 statistical software for statistical analysis, all patients before and after surgery JOA score, NDI score, VAS score were used to compare the double $\mathrm{t}$ test, $P<0.05$ was statistically significant. When encountering some data such as indeterminate results, missing data, and data outliers, we will delete these data.

\section{Results}

All patients' surgerys were successfully completed. Surgery time was $45 \mathrm{~min}-100$ min (the average time was $67.2 \pm 17.5 \mathrm{~min}$ ), intraoperative blood loss was 30 - 80 $\mathrm{ml}$ (the average blood loss was $61.2 \pm 16.3 \mathrm{ml}$ ). All patients after surgery neck pain, neck stiffness and other symptoms were improved in varying degrees.17 cases of elderly patients after 1 week of recurrence of cervical spine showed a good atlantoaxial reduction, dentate position fixed, iliac bone graft and pedicle screw position is good. The patients were followed up for 6 to 48 months (the average time was $27.4 \pm 12.4$ months). 16 cases of patients with good prognosis, no complications; 1 case of patients stitches when the neck wound does not heal the situation, the wound dressing and cleaning regularly, after 1 month the review of patients with cervical neck healing is complete heal, according to Frankel spinal cord injury nerve function classification [7]: D grade 3 cases were successfully restored to E level. During the follow-up procedure, there was no complication of that lantoaxial pedicle screw loosening, diversion and rupture, and there were no complications such as iliac bone grafting. Fracture healing is good, no odontoid fracture isn't healed or atlantoaxial joint instability, etc. (Figure 4 and Figure 5). Later follow-up data in patients with upper cervical spine are $30^{\circ}-60^{\circ}$ varying degrees of rotational function is limited, last follow-up scores of VAS were significantly lower than before surgery, JOA score and NDI score improved compared with preoperative, the specific data is summarized on Table 1.
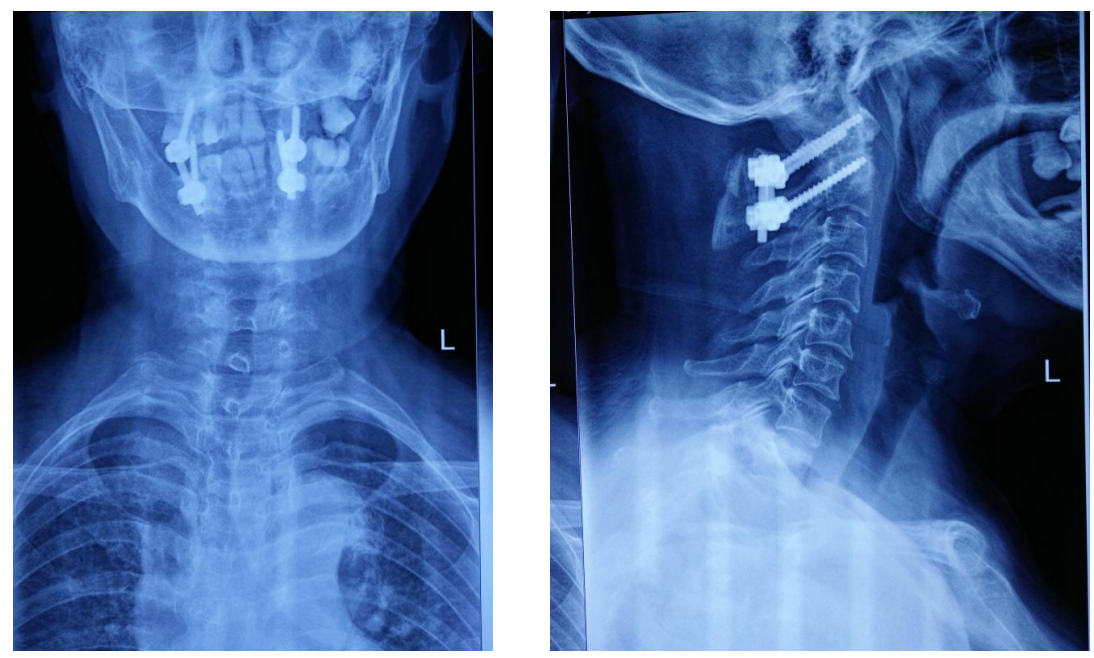

Figure 4. Postoperative review two months after operation, X-ray showed a good resection, atlantoaxial sequence integrity, iliac bone graft and internal fixation position is good, part of the fusion of bone graft, odontoid fracture line fuzzy. 

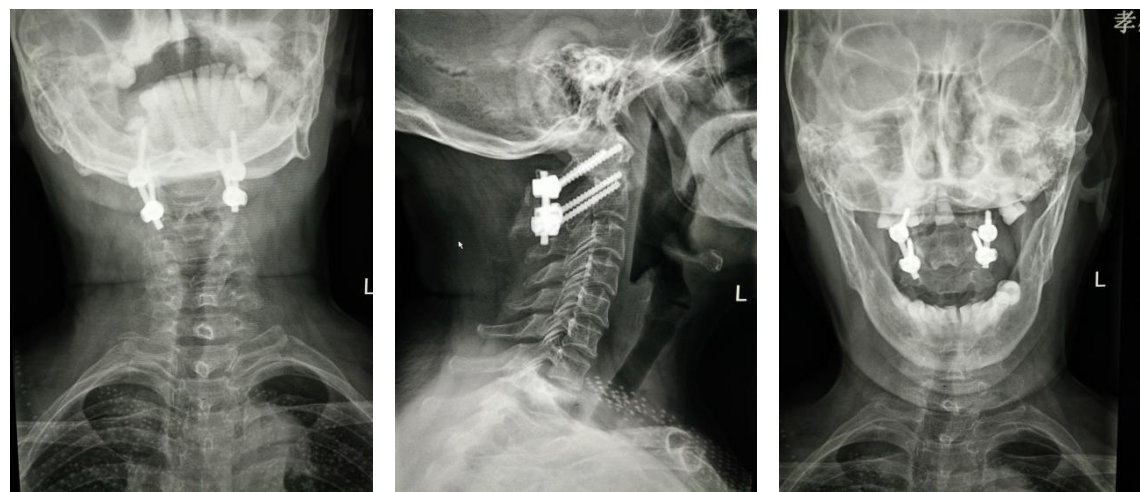

Figure 5. After six months of recurrence, the cervical vertebrae showed a good position in the pedicle screw, the atlantoaxial sequence was complete, the cervical vertebrae showed a good tooth profile, the fracture line was blurred, the fracture was healed. This patient has a good cervical flexion and extension and rotation function.

Table 1. 17 patients before and after surgery VAS, JOA, NDI score comparison $\bar{\chi} \pm \mathrm{s}$.

\begin{tabular}{cccc}
\hline Item & VAS & JOA & NDI \\
\hline Before surgery & $7 \pm 1.1$ & $10.7 \pm 1.3$ & $36.1 \pm 3.8$ \\
Last follow-up & $3.4 \pm 1.1$ & $15.5 \pm 1.2$ & $14.6 \pm 3.0$ \\
$t$ & 14.3 & 23.9 & 27.1 \\
$P$ & $<0.001$ & $<0.001$ & $<0.001$
\end{tabular}

\section{Discussion}

\subsection{Elderly Patients with Anderson II Odontoid Fractures Should Be Treated as Soon as Possible}

More than half of the rotational function of the cervical spine is borne by the activities of the atlantoaxial. The dentate process is the center of the atlantoaxial joint. The stability of the atlantoaxial joint depends on the integrity of the lateral ligament on both sides. The cruciate ligament surrounds the odontoid process, the dentate process before and after the synovial cavity, in order to facilitate the activities between the atlantoaxial function, in addition, atlas are connected with toe ligament ligament, and wing-shaped ligament, etc. These ligaments all around the dentate, together maintain the stability of the odontoid process and atlantoaxial. Odontoid dorsal side of the spinal cord, the base is weak, so when the trauma occurs, prone to fracture. According to Anderson-D'Alonzo classification, type II fractures can be divided into the following three categories: II a type (fracture line was horizontal direction); II b type (fracture line was obliquely forward oblique); II c type (Fracture line was on the back before the oblique line). Due to odontoid fracture easily damage the odontoid process nutrition vessels, and can lead to atlantoaxial joint instability, spinal cord damage, and even life-threatening [8]. Atlantoaxial central axis of structural loss caused by odontoid fracture, instability direction, will lead to varying degrees of atlantoaxial dislocation, displaced odontoid or atlas after the arch will constitute spinal cord compression, The reports [9] $33 \%$ to $65 \%$ of patients with odontoid fractures due to no obvious clinical symptoms and not timely effective diagnosis and treatment, gradually evolved 
into old odontoid fractures. So the type II odontoid fracture, the patient preoperative examination no obvious surgical contraindications, theoretically should be actively early diagnosis and treatment to facilitate the integrity of the dentate structure and its corresponding axis recovery, and maintain the atlantoaxial joint The stability of the corresponding function of cervical recovery [10].

\subsection{The Indications of Anderson Type II Odontoid Fracture in Elderly Patients by Posterior Pedicle Screw Fixation Combined with Iliac Bone Grafting}

In recent years, studies have shown that anterior screw fixation treatment of type II odontoid fractures, although trauma is small, better protection of the atlantoaxial joint mobility and other advantages [1] [11], but its main application in Anderson classification II A And II B-type odontoid fractures, the following situations need attention: 1) minor patients, especially young patients and odontoid more small type II fractures; 2) atlas after the bow height is less than $4 \mathrm{~mm}$ [12]; 3) type dentate Sudden comminuted fracture; 4) duration $>3$ months old type II fractures without surgical treatment, or combined with cruciate ligament rupture; 5) merger with Jefferson fracture type II fracture or merger with atlas lateral mass fractures. In recent years, studies [13] [14] have shown that there are many intraoperative and postoperative complications of anterior surgery, such as delayed healing or nonunion, anterior screw fixation, esophageal fistula, and so on. In addition to the above, other types of odontoid fractures can be treated with posterior pedicle screw fixation; at the same time, for the anterior screw fixation failure of type II odontoid fractures and the merger with neck stiffness symptoms of Anderson type II odontoid fractures, posterior pedicle screw fixation can be considered a good remedy [15] [16].

\subsection{Related Research on Autologous Iliac Bone Grafting}

The same to the general spine to laminectomy after the bone graft, Autologous iliac bone has been considered to be posterior pedicle screw fixation after bone fusion "golden standard". Although recent studies at home and abroad have shown that autologous iliac bone graft-related complications of $24 \%$ - 36\% [17] [18], and may cause increased intraoperative bleeding [19], but this group of patients were no significant complication were reported in the before literature. Autologous iliac bone graft was used in this group. The intraoperative blood loss did not increase significantly $(61.2 \pm 16.3 \mathrm{ml})$. All patients were followed up. There is no loose rod system loose or broken, review the imaging results are prompted patients with odontoid fracture to achieve bone healing, and internal fixation without loosening, fracture and so on.

\subsection{The Advantages of Posterior Pedicle Screw Fixation Combined with Iliac Bone Grafting}

Compared with the traditional posterior fixation technique of Galliel, Apofix laminectomy and Mgerl atlantoaxial screw, the surgical method proposed in this re- 
port is more reliable. And in the actual operation of the patient less invasive, implanted pedicle screw fast and easy, without frequent perspective to determine the location, and the possibility of long-term recurrence of cervical pain will greatly reduced, it can also obtain a better atlantoaxial joint biostation Sex [20].

\section{Conclusions and Some Notes on Surgical Operation}

1) During surgery, we should avoid damage to the venous sinus of atlas and the medial and C2 nerve root. Operation must be gentle, if necessary, using the brain cotton or gelatin sponge to protect the relevant organization, so that surgical field of vision is clear; 2) During surgery, in order to avoid damage to the vertebral artery and related important blood vessels, nerves, the current study is generally considered that both sides of the peel to reveal the width of the general should not exceed $15 \mathrm{~mm}$, so as not to peel off to the transverse process, accidentally damage the artery, resulting in bleeding, and even endangering the lives of patients. Atlas posterior pedicle screw fixation should be try to bias the side, to the opposite, the axis should be as far as possible on the side, so as not to damage important blood vessels and nerves.

Typical cases: The patient is 65 years old. Neck pain and activity are limited after injury for 13 hours.

\section{References}

[1] Anderson, L.D. and D'Alonzo, R.T. (1974) Fractures of the Odontoid Process of the Axis. The Journal of Bone and Joint Surgery, 56, 1663-1674. https://doi.org/10.2106/00004623-197456080-00017

[2] Huybregts, J.G., Jacobs, W.C. and Vleggeert-Lankamp, C.L. (2013) The Optimal Treatment of Type II and III Odontoid Fractures in the Elderly: A Systematic Review. European Spine Journal, 22, 1-13. https://doi.org/10.1007/s00586-012-2452-3

[3] Kim, S.K., Shin, J.J., Kim, T.H., et al. (2011) Clinical Outcomes of Halo-Vest Immobilization and Surgical Fusion of Odontoid Fractures. Journal of Korean Neurosurgical Society, 50, 17-22. https://doi.org/10.3340/jkns.2011.50.1.17

[4] Denaro, V., Papalia, R., Di Martino, A., et al. (2011) The Best Surgical Treatment for Type II Fractures of the Dens Is Still Controversial. Clinical Orthopaedics and Related Research, 469, 742-750. https://doi.org/10.1007/s11999-010-1677-x

[5] Grauer, J.N., Shafi, B., Hilibrand, A.S., et al. (2005) Proposal of a Modified, Treatment-Oriented Classification of Odontoid Fractures. The Spine Journal, 5, 123-129. https://doi.org/10.1016/j.spinee.2004.09.014

[6] David, G. and Andrew, S. (2002) ABC of Spinal Cord Injury. BMJ Publishing Group, London.

[7] Holds Worth, P. (1970) Fractures, Dislocations and Fractures-Dislocations of the Spine. The Journal of Bone and Joint Surgery, 52, 1534.

https://doi.org/10.2106/00004623-197052080-00002

[8] Dan, J., Zou, J., Shao, Y., et al. (2017) Treatment of Odontoid Fracture with Atlantoaxial Instability by Internal Fixation of Posterior Cruciate Bar System. Chinese Journal of Bone and Joint Injury, 32, 57-58.

[9] Tao, C., Ni, B., Wang, J., et al. (2006) Clinical Study of Delayed Odontoid Fracture Associated with Delayed Spinal Cord Injury. Chinese Journal of Orthopedics and 
Traumatology, 8, 1135-1138.

[10] Zhou, Z., Zhu, Y. and Xie, Y. (2013) Treatment of Traumatic Unstable Type II Odontoid Fracture with Posterior Screw Fixation. Journal of Clinical Orthopedics, 16, 605-607.

[11] Ren, H., Wang, J., Chen, J., et al. (2014) Treatment of Odontoid Fracture with Anterior Cervical Hollow Screw Fixation. Chinese Journal of Spine and Spinal Cord, 16, 817-819.

[12] Tan, M., Wang, H., Wang, Y., et al. (2003) Morphometrie Evaluation of Screw Fixation in Atlas via Posterior Arch and Lateral Mass. Spine, 28, 888-895. https://doi.org/10.1097/01.BRS.0000058719.48596.CC

[13] White, A., Hashinloto, R., Norveu, D.C., et al. (2010) Mor-Biditv and Mortalitv Related to Odontoid Fracture Surgery in the Elderly Population. Spine, 35, 146-157.

[14] Ma, X., Yin, Q., Xia, H., et al. (2011) Surgical Selection and Treatment of Fresh Type II Odontoid Fractures. Chinese Journal of Spine and Spinal Cord, 21, 550-553.

[15] Cui, X., Yu, H., Jiang, J., et al. (2016) Surgical Treatment of Odontoid Fracture with Atlantoaxial Instability. Chinese Journal of Spine and Spinal Cord, 26, 316-322.

[16] Guo, Q., Deng, Y., Wang, J., et al. (2016) Comparison of Clinical Outcomes of Posterior C1-C2 Temporary Fixation without Fusion and C1-C2 Fusion for Fresh Odontoid Fractures. Neurosurgery, 78, 77-82.

https://doi.org/10.1227/NEU.0000000000001006

[17] Scheerlinck, L.M., Muradin, M.S., van der Bilt, A., et al. (2013) Donor Site Complications in Bone Grafting: Comparison of Iliac Crest, Calvarial, and Mandibular Ramus Bone. The International Journal of Oral \& Maxillofacial Implants, 28, 222-227. https://doi.org/10.11607/jomi.2603

[18] Frisenbichler, J., Maurer-Ertl, W., Sadoghi, P., et al. (2014) Adverse Reactions of Artificial Bone Graft Substitutes: Lessons Learned from using Tricalcium Phosphate geneX. Clinical Orthopaedics and Related Research, 472, 976-982. https://doi.org/10.1007/s11999-013-3305-Z

[19] Liao, Y., Tang, Q., Li, G., et al. (2016) Clinical Observation of Pedicle Screw Fixation in the Treatment of Fresh Odontoid Fractures in Elderly Patients. Guangdong Medical, 37, 3046-3048.

[20] Ma, W., Liu, G., Xu, R., et al. (2010) Treatment of Traumatic Upper Cervical Spine with Atlas Pedicle Screw and Pedicle Screw Fixation. Chinese Journal of Spine and Spinal Cord, 20, 214-218. 